
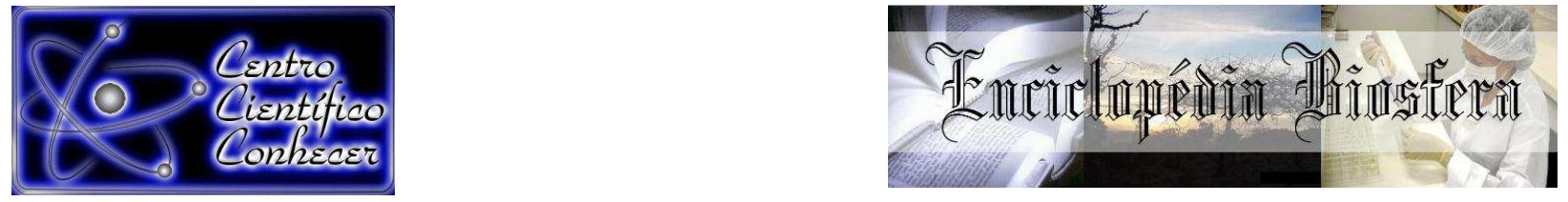

\title{
BIOMETRIA E ASPECTOS FISIOLÓGICOS DE FRUTOS E SEMENTES DE Dulacia candida (POEPP.) KUNTZE (OLACACEAE)
}

Ednéia Araújo dos Santos ${ }^{1}$, Romário de Mesquita Pinheiro ${ }^{2}$, Evandro José Linhares Ferreira $^{3}$, Marilene de Campos Almeida ${ }^{4}$

1. Engenheira Florestal, Mestre em Botânica, Instituto Nacional de Pesquisas da Amazônia - INPA, Rio Branco, Acre (edneiasantos_14@hotmail.com).

2. Graduando em Engenharia Agronômica, Universidade Federal do Acre, Instituto Nacional de Pesquisas da Amazônia - INPA, Rio Branco, Acre.

3. Engenheiro Agrônomo, Doutor em Botânica, Pesquisador do Instituto Nacional de Pesquisas da Amazônia - INPA, Rio Branco, Acre.

4. Engenheira Agrônoma, Doutora em Biologia Vegetal, Laboratório de Sementes Florestais do Parque Zoobotânico da UFAC, Rio Branco, Acre.

Recebido em: 08/09/2015 - Aprovado em: 14/11/2015 - Publicado em: 01/12/2015 DOI: http://dx.doi.org/10.18677/Enciclopedia_Biosfera_2015_102

\begin{abstract}
RESUMO
Dulacia candida (Poepp.) Kuntze (Olacaceae) é uma espécie arborescente com ampla distribuição na América do Sul. No Brasil ocorre nas regiões Norte, Nordeste e Centro-Oeste e apesar de sua extensa distribuição, é uma espécie pouco estudada, condição comum para numerosas espécies florestais nativas do país. Este estudo avaliou as características biométricas dos frutos e sementes, e a sensibilidade das sementes à dessecação utilizando o método SCR. Os frutos usados eram oriundos de florestas de várzea das margens do Riozinho do Andirá, em Sena Madureira, Acre (0939'50,6"S; 6802'54,5"W ). Na avaliação biométrica foram utilizados 200 frutos medidos e pesados com o auxílio de paquímetro e balança eletrônica. Dos frutos foram avaliados a massa total, comprimento, diâmetro e profundidade da polpa, e das sementes a massa, comprimento e diâmetro. Foi avaliada também a massa do endosperma e do tegumento. Para todas as variáveis avaliadas foram calculados, com o auxílio do programa estatístico BioEstat 5.0, os valores máximo e mínimo, média $(\mathrm{m})$, desvio padrão (DP), coeficiente de variação (CV) e o coeficiente de correlação de Pearson (r). A previsão da sensibilidade à dessecação (P) utilizou a metodologia SCR. Os resultados obtidos indicam que a massa da polpa e das sementes está diretamente relacionada com a massa dos frutos, ou seja, frutos mais pesados apresentam maior massa de polpa e de sementes. As sementes possuem tolerância à dessecação, indicando que as mesmas são do tipo ortodoxa.
\end{abstract}

PALAVRAS-CHAVE: Amazônia, dessecação, ortodoxas. 


\title{
BIOMETRICS AND PHYSIOLOGICAL ASPECTS OF FRUIT AND SEEDS OF Dulacia candida (Poepp.) KUNTZE (OLACACEAE)
}

\begin{abstract}
Dulacia candida (Poepp.) Kuntze (Olacaceae) is a tree species with wide distribution in South America. It is found in the North, Northeast and Midwest regions of Brazil, and despite its wide distribution, is poorly studied, a common condition for numerous forest species native to the country. This study evaluated the biometric characteristics of fruits and seeds, and the sensitivity of seeds to desiccation using the SCR method. The fruits used were collected in várzea forests of the banks of the Riozinho Andirá in Sena Madureira, Acre (0939'50,6 "S, 6802'54,5"W). For the biometric evaluations 200 fruits were measured and weighted with the aid of a caliper and an electronic scale. The fruits were evaluated for their total mass, length, diameter and pulp depth, and the seeds for their mass, length and diameter. It was also evaluated the endosperm mass and the seed coat. The calculations of the maximum and minimum values, average $(\mathrm{m})$, standard deviation (SD), coefficient of variation $(\mathrm{CV})$ and the Pearson correlation coefficient $(r)$ were done with the aid of the statistical program BioEstat 5.0. The prediction of sensitivity to desiccation (P) used the SCR methodology. The results indicate that the mass of the pulp and the seeds is directly related to the average fruit weight, i.e., heavier fruits have higher pulp and seeds mass. The seeds are desiccation tolerant, indicating that they are of the orthodox type.
\end{abstract}

KEYWORDS: Amazon, native species, Sena Madureira

\section{INTRODUÇÃO}

A família Olacaceae apresenta distribuição pantropical e inclui 24 gêneros, sendo12 monotípicos, e cerca de 150 espécies. No Brasil ocorrem 12 gêneros e aproximadamente 60 espécies mais frequentes na região amazônica (SOUZA \& LORENZI, 2012). Dentre os gêneros de Olacaceae, Dulacia, com cerca de 14 espécies, ocorre na América do Sul e está distribuída no Brasil (Acre, Amazonas, Rondônia, Maranhão, Mato Grosso, Rio de Janeiro e Distrito Federal), Guianas, Venezuela, Colômbia, Equador, Peru e Bolívia (MONACHINO, 1961; NICKRENT et al., 2010; GRANDTNER \& CHEVRETTE, 2013).

As espécies de Dulacia apresentam frutos ovoides ou elipsoides, o pericarpo é fino e carnoso, geralmente alaranjado ou avermelhado, e são dispersos por macacos e outros animais (RIBEIRO et al., 1999; SOUZA \& LORENZI, 2012). Segundo RIBEIRO et al. (1999), os frutos de Dulacia guianensis (Engl.) Kuntze são comestíveis e ricos em gordura, sendo utilizados para o preparo de sopa por populações tradicionais. Conhecida popularmente como cajazinho da várzea, Dulacia candida (Poepp.) Kuntze é uma espécie arborescente que atinge entre 3 e 12 metros de altura.

A espécie ocorre nas regiões norte, nordeste e centro-oeste do Brasil, além de Venezuela, Colômbia, Equador, Peru e Bolívia, tanto em florestas de terra firme como em campinaranas, várzeas e igapós e em áreas alteradas. Seu tronco é mais largo na base e o ritidoma é rugoso e de coloração marrom acinzentado. Os botões florais são esverdeados e suas flores desenvolvidas exibem pedicelo verde, sépalas carnosas branco-esverdeadas, pétalas brancas, filetes e estiletes brancos e anteras amarelas. Seu fruto é drupáceo, de cor verde quando imaturo, tornando-se 
amarelado ou alaranjado quando maduro (RIBEIRO et al., 1999; SOUZA \& LORENZI, 2012; HERBÁRIO INPA, 2015).

Embora seja uma espécie com ampla distribuição na América do Sul, são poucas as informações técnico-científicas sobre $D$. candida, em sua maioria restrita a descrições morfológicas e de ocorrência. Esta situação é comum a um grande número de espécies florestais amazônicas, quase todas desconhecidas quanto às características biométricas de frutos e sementes, bem como a respeito da tolerância de suas sementes à dessecação e o comportamento durante o armazenamento.

Estudos biométricos fornecem informações úteis para a conservação e a exploração racional de recursos naturais de valor econômico, como frutos e sementes, fornecem informações que auxiliam na distinção entre espécies do mesmo gênero, e são relevantes para a detecção de variabilidade genética entre populações durante o desenvolvimento de programas de melhoramento genético (CRUZ et al., 2001; BATTILANI et al., 2011; CHRISTRO et al., 2012; FERREIRA et al., 2012; ROCHA et al., 2014).

Atualmente as sementes são classificadas em três categorias quanto ao seu comportamento fisiológico no armazenamento: sementes ortodoxas, que toleram dessecação a baixos conteúdos de água (2\%-5\%) e podem ser armazenadas em baixas temperaturas $\left(-20^{\circ} \mathrm{C}\right)$, condições que maximizam 0 tempo de armazenamento. As sementes intermediárias, que não toleram a dessecação a baixos conteúdos de água (10\%-12 \%), mas que podem ser armazenadas a baixas temperaturas (geralmente acima de $0^{\circ} \mathrm{C}$ ); e sementes recalcitrantes, comuns entre as espécies florestais da região tropical, as quais não toleram dessecação a baixos conteúdos de água ( $<12 \%$ ), nem 0 armazenamento a baixas temperaturas (SACANDÉ et al., 2004; NERY et al., 2014; PRITCHARD et al., 2014).

O conhecimento sobre a sensibilidade à dessecação das sementes de espécies florestais é importante porque para determinar a melhor forma de armazenamento das mesmas (SCALON et al., 2012; SILVA et al., 2012; NERY et al., 2014; JOSHI et al., 2015), e são escassas para grande maioria das espécies de árvores no mundo (PRITCHARD et al, 2014. Nesse contexto, e visando contribuir com estudos de armazenamento de sementes, DAWS et al. (2006) estudaram o comportamento de 104 espécies com relação à sensibilidade à dissecação e concluíram que existe uma tendência para que espécies com maior sensibilidade a dessecação apresentem maior razão entre o envoltório da semente e a massa da semente, "Seed Coat Ratio"-SCR, metodologia rápida e pratica descrita por PRITCHARD et al. (2004) para pré-classificar as sementes para fins de armazenamento.

Diante do exposto, o objetivo deste estudo foi caracterizar biometricamente os frutos e sementes e pré-classificação quanto à sensibilidade à dessecação das sementes de $D$. candida utilizando-se o método SCR.

\section{MATERIAL E MÉTODOS}

Os frutos usados neste estudo foram colhidos de plantas existentes nas margens do Riozinho do Andirá, no Município de Sena Madureira, Acre (0939'50,6"S; 6802'54,5"W), em março de 2015, em uma área de floresta de várzea sujeita a inundações periódicas. Os frutos foram levados para o Laboratório de Sementes Florestais do Parque Zoobotânico (PZ) da Universidade Federal do Acre (UFAC), em Rio Branco, Acre, onde foi realizada a avaliação biométrica. 
Para essa avalição, foram escolhidos 200 frutos maduros, sem deformações e sadios. Os frutos e as sementes foram pesados em balança digital com precisão de $0,001 \mathrm{~g}$ e as medidas de comprimento $(\mathrm{mm})$ e diâmetro $(\mathrm{mm})$ com paquímetro digital de precisão de $0,01 \mathrm{~mm}$. Nos frutos foram avaliados a massa total, comprimento, diâmetro e profundidade da polpa. Depois de medidos, os frutos foram despolpados e as sementes resultantes avaliadas quanto à massa, comprimento e diâmetro. Foi avaliada também a massa do endosperma e do tegumento. $O$ valor referente à massa da polpa foi obtido pelo cálculo da diferença entre a massa total do fruto e a massa da semente de acordo com metodologia utilizada por CARVALHO \& MÜLLER, (2005).

Para todas as variáveis avaliadas foram calculados os valores máximo e mínimo, média (m), desvio padrão (DP), coeficiente de variação (CV) e o coeficiente de correlação de Pearson ( $r$ ). Este coeficiente varia de $-1 a+1$ e quanto mais próximo desses valores, mais forte é a correlação entre as variáveis examinadas (AYRES et al., 2007). A estatística descritiva e o coeficiente de correlação de Pearson foram calculados com o auxílio do programa estatístico BioEstat 5.0.

Para a previsão da tolerância ou intolerância ao dessecamento das sementes, foram utilizadas quatro repetições de 50 unidades de dispersão as quais foram dissecadas em: endocarpo + testa (envoltório) e eixo embrionário + cotilédones e endosperma (semente). O material foi pesado, envolto em papel alumínio e mantido em estufa elétrica a $105^{\circ} \mathrm{C} \pm 3$, por 24 horas. Três pesagens subsequentes foram realizadas até a estabilização do peso.

Posteriormente, a massa de cada parte dessecada foi determinada em uma balança de precisão. Para o cálculo da relação entre o envoltório e a massa da semente (Seed Coat Ratio-SCR) foi utilizada a seguinte equação: SCR = peso seco do envoltório (endocarpo + testa) / peso seco da unidade de dispersão integral. Para o calculo da probabilidade da semente ser recalcitrante foi utilizada a seguinte equação, de acordo com DAWS et al. (2006):

$$
P=\frac{e^{3.269-9.974 a+2.156 b}}{1+e^{3.269-9.974 a+2.156 b}}
$$

Sendo:

$\mathrm{a}=\mathrm{SCR}$

$\mathrm{b}=$ massa da semente

Assim, se $\mathrm{P}<0,5$ a semente é provavelmente tolerante a dessecação, podendo ser classificada como ortodoxa; se $\mathrm{P}>0,5$ semente é provavelmente sensível à dessecação, podendo ser classificada como recalcitrante.

\section{RESULTADOS E DISCUSSÃO}

Na tabela 1 e nas figuras 2, 3 e 4 são apresentados os dados das avaliações biométricas dos frutos, sementes, endospermas e tegumentos de Dulacia candida. Seus frutos possuem formato oblongo-obovado (Figura 1) e a maioria apresentou massa entre $3,21 \mathrm{~g}$ e 4,40 $\mathrm{g}(71 \%)$, comprimento entre $21,41 \mathrm{~mm}$ e 25,80 mm $(86,5 \%)$, diâmetro entre $14,21 \mathrm{~mm}$ e $17,40 \mathrm{~mm}(86 \%)$, profundidade da polpa de $0.40 \mathrm{~mm}(62,5 \%)$ e massa da polpa entre $2,41 \mathrm{~g}$ e 3,60 g (75,5\%). Foi possível notar que os frutos com maior massa foram também os mais compridos, com maiores diâmetro e profundidade da polpa (Figura 2 A-E). 


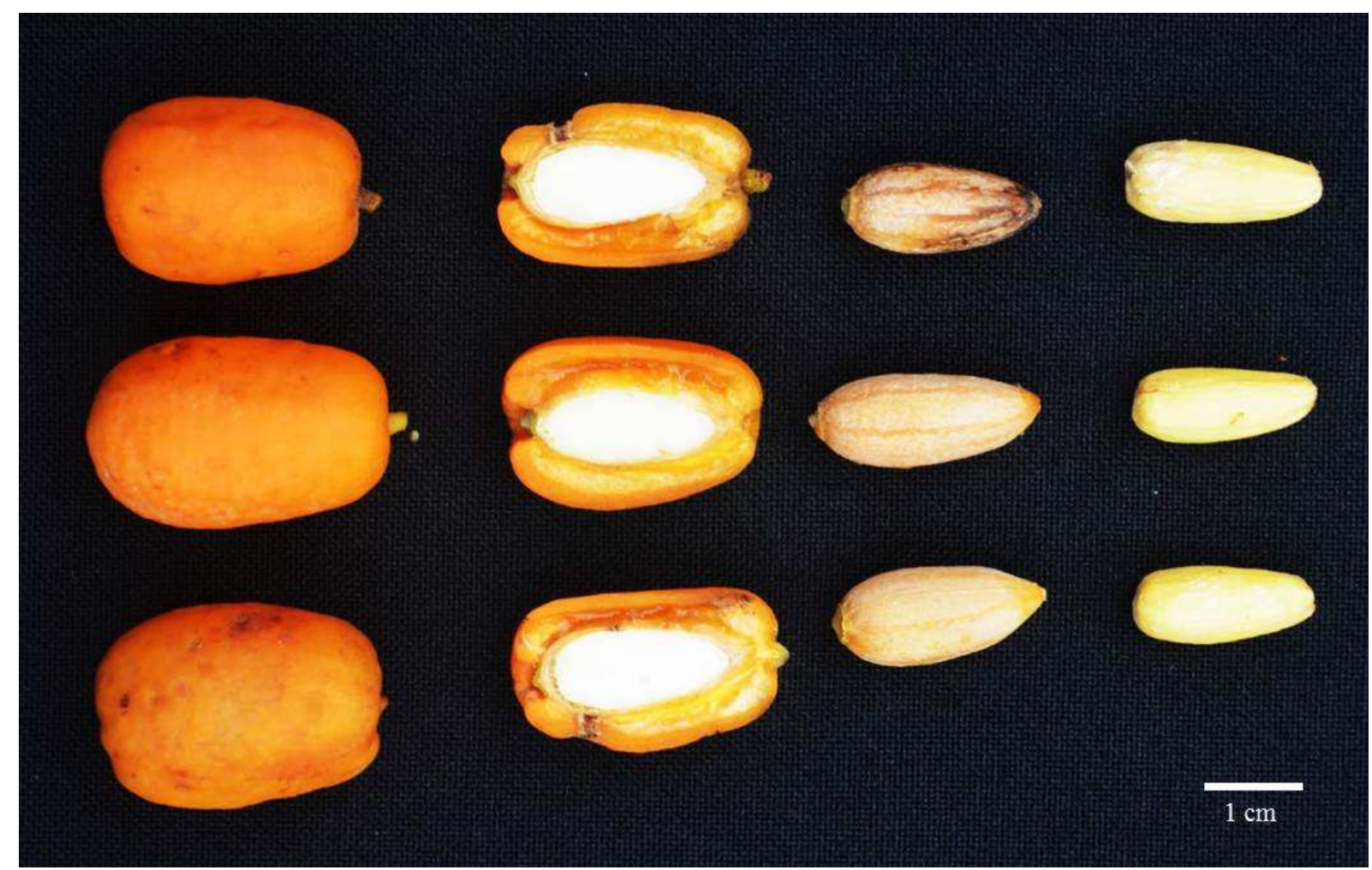

FIGURA 1 - Frutos, sementes e endosperma de Dulacia candida ocorrentes na região de Sena Madureira, Acre. Foto: Evandro J. L. Ferreira.

TABELA 1 - Valores das variáveis avaliadas dos frutos e sementes de Dulacia candida oriundos de Sena Madureira, Acre. DP=desvio padrão, $\mathrm{CV}=$ coeficiente de variação.

\begin{tabular}{lccccc}
\hline Características biométricas & Mínimo & Máximo & Média & DP & CV (\%) \\
\hline Fruto & & & & & \\
\hline Massa $(\mathrm{g})$ & 2,09 & 5,59 & 3,84 & 0,59 & 15,50 \\
Comprimento $(\mathrm{mm})$ & 17,40 & 27,20 & 23,49 & 1,45 & 6,19 \\
Diâmetro (mm) & 11,80 & 18,70 & 15,64 & 1,18 & 7,55 \\
Profundidade da polpa (mm) & 0,30 & 0,50 & 0,40 & 0,06 & 15,24 \\
Massa da polpa (g) & 1,89 & 4,61 & 3,12 & 0,49 & 15,66 \\
\% Polpa & 75,18 & 93,95 & 81,37 & 2,78 & 3,42 \\
\hline Semente & & & & & \\
\hline Massa (g) & 0,16 & 0,99 & 0,72 & 0,15 & 20,59 \\
Comprimento (mm) & 16,10 & 22,60 & 19,74 & 1,20 & 6,07 \\
Diâmetro (mm) & 6,70 & 8,70 & 7,86 & 0,40 & 5,10 \\
\% Semente & 6,05 & 24,82 & 18,63 & 2,78 & 14,92 \\
\hline Endosperma & & & & & \\
\hline Massa fresa (g) & 0,03 & 0,70 & 0,40 & 0,09 & 24,17 \\
Massa seca (g) & 0,01 & 0,51 & 0,33 & 0,09 & 26,85 \\
Comprimento (mm) & 5,10 & 17,80 & 15,14 & 1,98 & 13,07 \\
Diâmetro (mm) & 1,40 & 7,60 & 6,17 & 0,94 & 15,20 \\
\hline Tegumento & & & & & \\
\hline Massa fresca (g) & 0,12 & 0,41 & 0,20 & 0,03 & 15,45 \\
Massa seca $(\mathrm{g})$ & 0,10 & 0,28 & 0,17 & 0,03 & 14,58 \\
\hline
\end{tabular}


Não foram identificados outros estudos abordando a biometria de frutos e sementes de $D$. candida, entretanto, foi possível avaliar amostras de herbários contendo frutos. No Brasil, o espécimen colhido por Schatz et al. 885 em floresta ripária ao longo do rio Mearim, no Maranhão (Depositado no herbário NY), apresenta frutos com comprimento variando entre 1,2 e 1,4 cm e diâmetro entre 1,1 e 1,2 cm. Em floresta primária do Departamento de Loreto, Peru, a amostra botânica colhida por Rodolfo Vásquez e G. Criollo n 7.064 (Deposita da no Herbário NY) apresenta frutos com 1,8-2,0 cm de comprimento e 0,8 e 1,0 cm de diâmetro. Ambas as amostras apresentam frutos com dimensões inferiores aos avaliados no presente estudo. Isto não é incomum, pois, conforme sugere BOTEZELLI et al. (2000), indivíduos de uma mesma espécie estão sujeitos às variações de temperatura, pluviosidade, duração do dia e outras variáveis que, em algumas localidades, favorecem a expressão de algumas de suas características genéticas em detrimento de outras. GUSMÃO et al. (2006) creditaram as diferenças na massa da matéria fresca dos frutos de Byrsonima verbascifolia oriundos do Distrito Federal e de Minas Gerais às diferenças fenotípicas resultante da influência de fatores ambientais decorrentes das distintas localidades geográficas de origem das amostras.
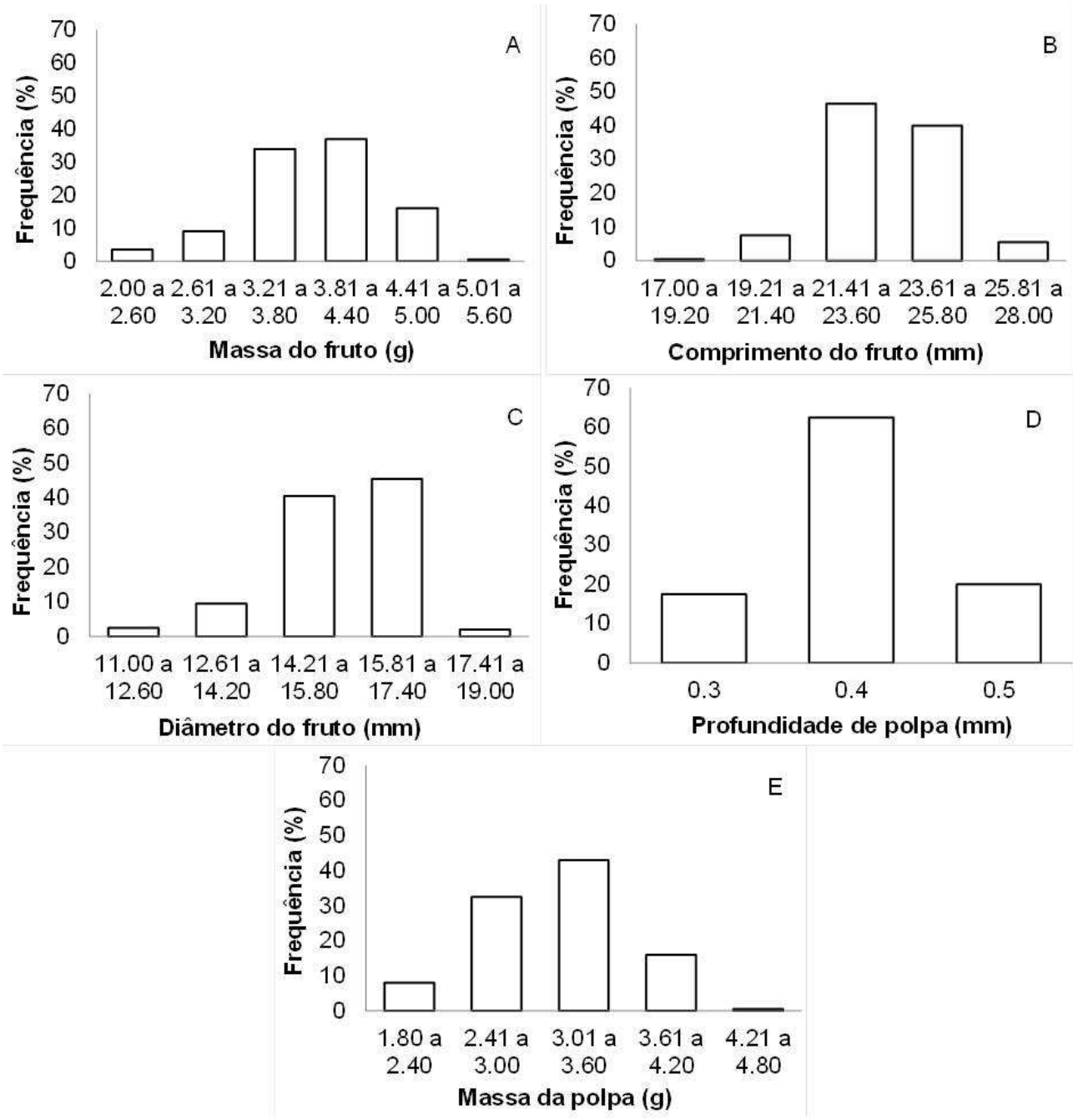

FIGURA 2 - Frequência (\%) da massa do fruto (A), comprimento (B), diâmetro dos frutos (C), Profundidade da polpa (D) e massa da polpa (E) de Dulacia candida oriundos de Sena Madureira, Acre. 
A maioria das sementes apresentou massa entre $0,56 \mathrm{~g}$ e $0,85 \mathrm{~g}(78 \%)$, comprimento entre $18,81 \mathrm{~mm}$ e $21,60 \mathrm{~mm}(74,5 \%)$ e diâmetro entre $7,21 \mathrm{~mm}$ e 8,40 $\mathrm{mm}(84,5 \%)$ (Figura $3 \mathrm{~A}-\mathrm{C}$ ). As sementes com maior massa também foram as com maiores comprimento e diâmetro. A importância de se conhecer a variação no tamanho das sementes é apontada por alguns estudos que sugerem que essa variável pode ou não influenciar em alguns aspectos da germinação das espécies. Por exemplo, SILVA et al. (2010) encontraram que o percentual de germinação de Artocarpus heterophyllus Lam. (Moraceae) foi influenciado pelo tamanho da semente que, no entanto, não influenciou a velocidade de germinação. SOCOLOWSKI et al. (2011) encontraram que o percentual de germinação de Tecoma stans (L.) Juss. ex Kunth (Bignoniaceae) foi maior em sementes com maior massa. Já DRESCH et al. (2013) encontraram que o percentual de germinação foi maior em sementes provenientes de frutos pequenos e menor em sementes oriundas de frutos grandes de Campomanesia adamantium (Cambess.) O. Berg (Myrtaceae).

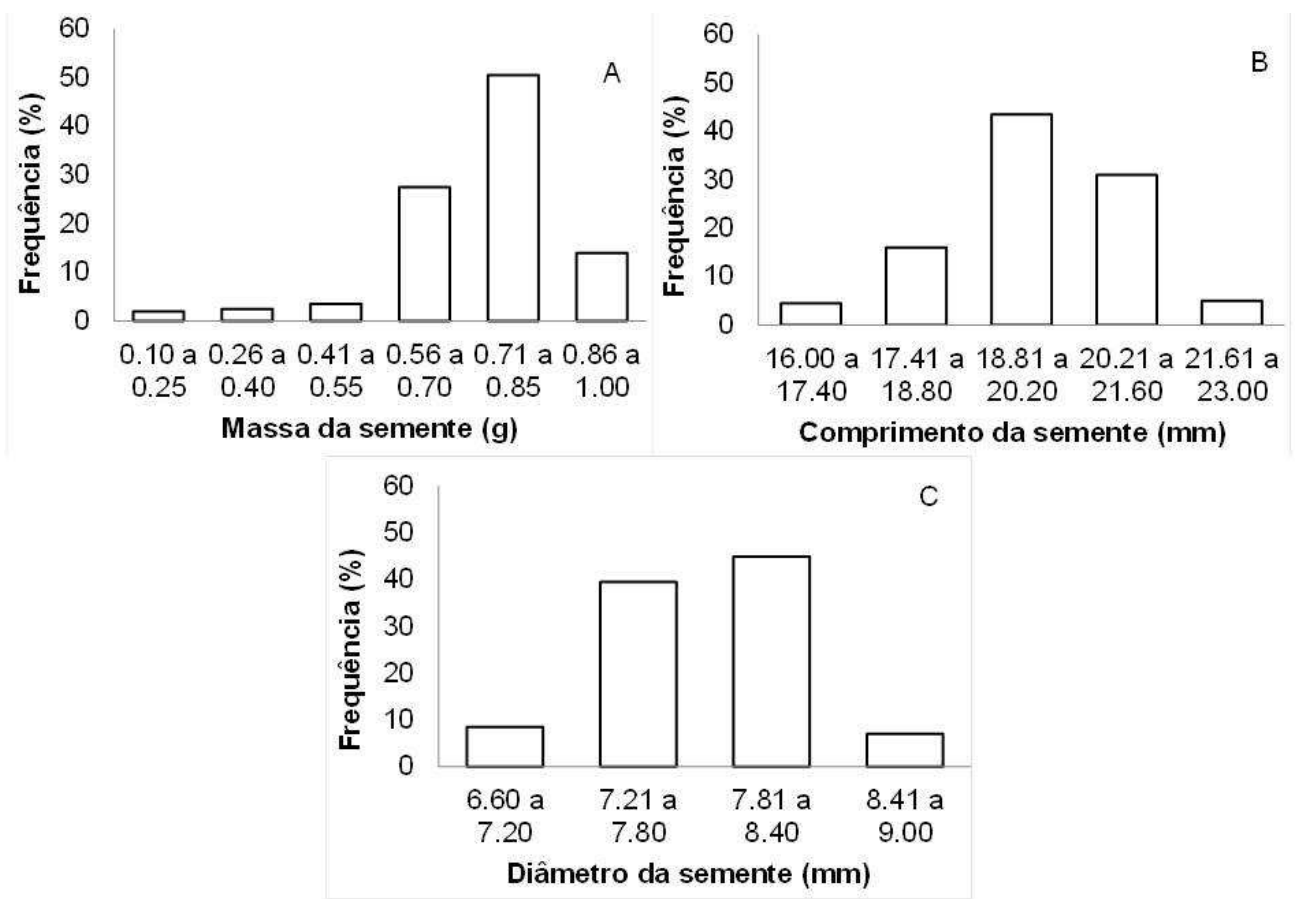

FIGURA 3 - Frequência (\%) da massa (A), comprimento (B) e diâmetro (C) das sementes de Dulacia candida oriundas de Sena Madureira, Acre.

A maioria dos endospermas apresentou massa fresca entre $0,34 \mathrm{~g} \mathrm{e} 0,49 \mathrm{~g}$ (75\%), comprimento entre $15,41 \mathrm{~mm}$ e $18,00 \mathrm{~mm}(54,0 \%)$, diâmetro entre $5,15 \mathrm{~mm}$ e $7,70 \mathrm{~mm}(93 \%)$, e a massa fresca do tegumento entre $0,19 \mathrm{~g} \mathrm{e} 0,24 \mathrm{~g}(64,5 \%)$ (Figura 4 A-D).

Observou-se que o coeficiente de variação (CV) foi maior nas medidas de massa dos frutos, polpa sementes e endosperma. Embora essa variabilidade não tenha sido testada, ela pode indicar alta variabilidade genética da espécie na área de coleta das sementes (GONÇALVES et al., 2013) ou influência de fatores ambientais TABARELLI et al. (2003). 

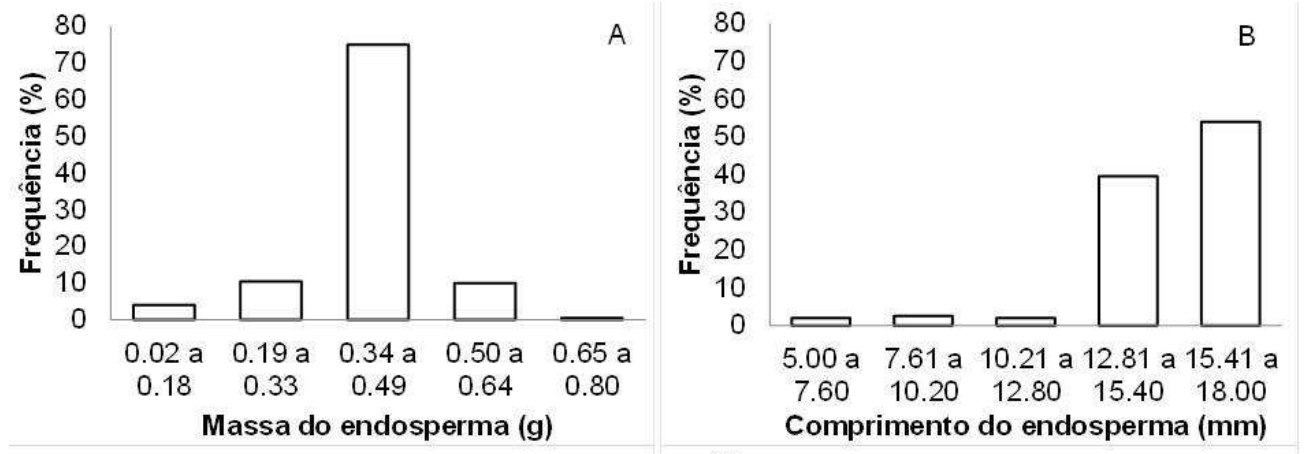

$\begin{array}{lllll}7.60 & 10.20 & 12.80 & 15.40 & 18.00\end{array}$

Comprimento do endosperma (mm)
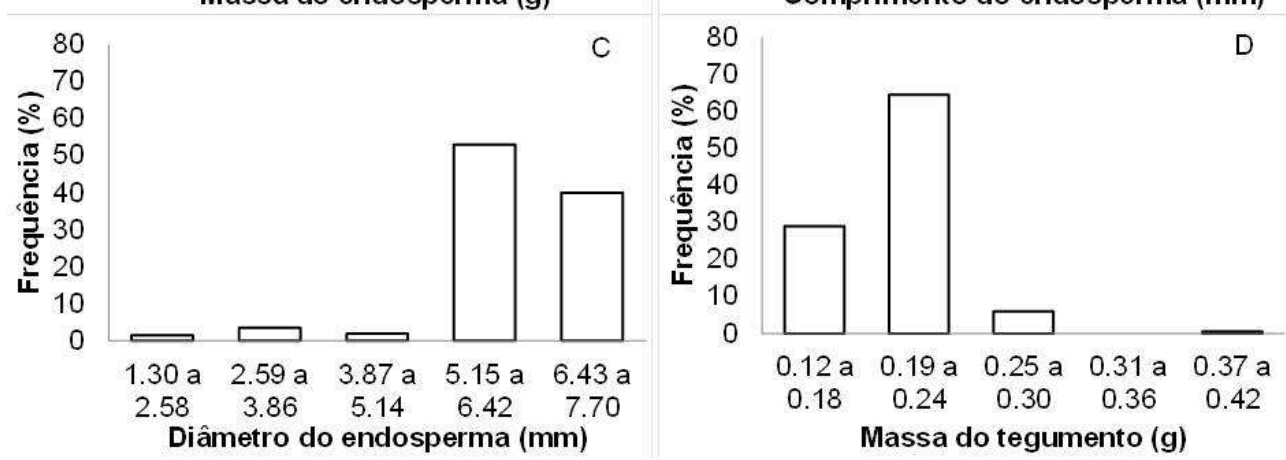

FIGURA 4 - Frequência (\%) da massa (A), comprimento (B) e diâmetro do endosperma (C) e massa do tegumento (D) de Dulacia candida oriundas de Sena Madureira, Acre.

Foi encontrada correlação de Pearson forte e positiva entre a massa do fruto e a massa da polpa $(0,9819)$, indicando que a massa do fruto está diretamente relacionada à massa da polpa. MOURA et al. (2010), PEDRON et al. (2004) e GONÇALVES et al. (2013) também observaram essa situação em seus estudos biométricos de frutos e sementes da palmeira Butia capitata (Mart.) Beccari e da mangaba (Hancornia speciosa Gomes). A correlação foi moderada e positiva entre a massa do fruto e a massa da semente $(0,7795)$, e entre a massa e o comprimento $(0,6922)$ e o diâmetro $(0,6922)$ do fruto. A correlação foi fraca e positiva entre a profundidade da polpa e o diâmetro da semente $(0,2333)$ (Tabela 2$)$.

TABELA 2 - Correlação de Pearson ( $r$ ) para as variáveis biométricas dos frutos e sementes de Dulacia candida avaliadas no Laboratório de Sementes Florestais do Parque Zoobotânico da UFAC, em Rio Branco, Acre. MF - Massa do fruto; CF - Comprimento do fruto; DF - Diâmetro do fruto; MP - Massa da polpa; PP - Profundidade da polpa; MS - Massa da semente; CS - Comprimento da semente; DS - Diâmetro da semente.

\begin{tabular}{ccccccccc}
\hline & MF & CF & DF & MP & PP & MS & CS & DS \\
\hline MF & & & & & & & & \\
CF & 0,6922 & & & & & & \\
DF & 0,6247 & 0.5515 & & & & & \\
MP & 0,9819 & 0.1610 & 0.6159 & & & & \\
PP & 0.5817 & 0,4300 & 0,5419 & 0.5800 & & & \\
MS & 0.7795 & 0.1438 & 0.4788 & 0.6470 & 0.4243 & & \\
CS & 0.7019 & 0.2438 & 0.3613 & 0.6990 & 0.4109 & 0.5148 & & \\
DS & 0.5463 & 0.0443 & 0.3125 & 0.4983 & 0,2333 & 0,5522 & 0.5515 \\
\hline
\end{tabular}


As sementes frescas de $D$. candida pesam em média $0,72 \mathrm{~g}$ (Tabela 1), sendo que o endosperma representa $55 \%$ da massa total da semente, e o percentual restante refere-se aos tecidos envoltórios. A predição da sensibilidade à dessecação $(P)$ das sementes de $D$. candida foi de 0,02 , indicando que essa espécie é provavelmente tolerante à dessecação e, assim, classificada como ortodoxa. Esse resultado corrobora resultados de estudos feitos com outras espécies de Olacaceae, como Olax subscorpioides Oliv., Ximenia americana var. microphylla Welw. ex Oliver e Ximenia americana L., consideradas tolerantes à dessecação (ROYAL BOTANIC GARDENS KEW, 2015). Sabe-se que diversos mecanismos e processos fisiológicos durante a fase de maturação dos frutos regulam a longevidade e a aquisição ou perda da tolerância à dessecação (NERY et al., 2014). Estudos recentes revelam que o estádio de maturação das sementes pode resultar em acentuada redução na capacidade de armazenamento das sementes ortodoxas, assim como 0 nível de secagem das sementes antes do armazenamento (SANTANA, 2007). GOLD \& HAY (2014) ressaltam que a maioria das sementes ortodoxas adquire completa tolerância à dessecação pouco antes da dispersão natural. Assim, muito resta a elucidar a respeito dos mecanismos interligados que atuam sobre o comportamento ecofisiológico das sementes.

\section{CONCLUSÕES}

Frutos e sementes de Dulacia candida apresentam grande variação biométrica, indicando que a espécie apresenta alta variabilidade dessas características. As sementes apresentam baixa sensibilidade à dessecação, sendo pré-classificadas como ortodoxas.

\section{AGRADECIMENTOS}

Agradecemos aos técnicos do Laboratório de Sementes Florestais do Parque Zoobotânico da Universidade Federal do Acre pela ajuda na coleta dos frutos e disponibilização do espaço físico e dos materiais do laboratório para a realização desse estudo.

\section{REFERÊNCIAS}

AYRES, M.; AYRES JUNIOR, M.; AYRES, D. L.; SANTOS, A. A. S. Biostat 5.0: aplicações estatísticas nas áreas das ciências biológicas e médicas. Brasília: Sociedade Civil Mamirauá-Belém (MCT-CNPq), Belém. 2007. 364p. 1 CD-ROM.

BATTILANI, J. L.; SANTIAGO, E. F.; DIAS, E. S. Morfologia de frutos, sementes, plântulas e plantas jovens de Guibourtia hymenifolia (Moric.) J. Leonard (Fabaceae). Revista Árvore, Viçosa-MG, v. 35, n. 5, p.1089-1098, 2011.

BOTEZELLI, L.; DAVIDE, A. C.; MALAVASI, M. M. Características dos frutos e sementes de quatro procedências de Dipteryx alata Vogel (Baru). Cerne, Lavras, v. 6, p. 9-18, 2000.

CARVALHO, J. E. U.; MÜLLER, C. H. Biometria e rendimento percentual de polpa de frutas nativas da Amazônia. Comunicado Técnico 139, Belém: Embrapa Amazônia Oriental. 3 p. 2005. 
CHRISTRO, L. F.; AMARAL, J. F. T. do; LAVIOLA, B. G.; MARTINS, L. D.; AMARAL, C. F. Biometric analysis of seeds of genotypes of physic nut (Jatropha curcas L.). Agropecuária Científica no Semiárido, Campina Grande-PB, v. 8, n. 1, p. 01-03, 2012.

CRUZ, E. D.; MARTINS, F. O.; CARVALHO, J. E. U. Biometria de frutos e sementes e germinação de jatobá-curuba (Hymenaea intermedia Ducke), Leguminosae Caesalpinioideae. Revista Brasileira de Botânica, São Paulo-SP, v. 24, n. 2, p.161165, 2001.

DRESCH, D. M.; SCALON, S. de P. Q.; MASETTO, T. E.; VIEIRA, M. do C. Germinação e vigor de sementes de gabiroba em função do tamanho do fruto e semente. Pesquisa Agropecuária Tropical, Goiânia, v. 43, n. 3, p. 262-271. 2013.

DAWS, M. I.; GARWOOD, N. C.; PRITCHARD, H. W. Prediction of desiccation sensitivity in seeds of woody species: a probabilistic model based on two seed traits and 104 species. Annals of Botany, New York, USA, v.97, p. 667-674, 2006.

FERREIRA, C. D.; SOUTO, P. C.; LÚCIO, A. M. F. N.; SOUTO, J. S.; SOUZA, B. V. de. Avaliações biométricas e germinação de sementes de Coaçu (Triplaris surinamensis Cham). Revista Brasileira de Tecnologia Aplicada nas Ciências Agrárias, Guarapuava-PR, v. 5, n.1, p.147-162, 2012.

GOLD, K.; HAY, F. Identifying desiccation-sensitive seeds. Technical Information sheed n. 10. Millennium Seed Bank; Project Royal Botanical Gardens, Kew. 2014.

GONÇALVES, L. G. V.; ANDRADE, F. R.; MARIMON JUNIOR, B. R.; SCHOSSLER, T. R.; LENZA, E.; MARIMON, B. S. Biometria de frutos e sementes de mangaba (Hancornia speciosa Gomes) em vegetação natural na região leste de mato Grosso, Brasil. Revista de Ciências Agrárias, Lisboa, v. 36, n. 1, p. 31-40, 2013.

GRANDTNER, M. M.; CHEVRETTE, J. Dictionary of Trees, South America: Nomenclature, Taxonomy and Ecology. Quebec: Academic Press, 2013, v. 2, 1172p.

GUSMÃO, E.; VIEIRA, F. A.; FONSECA, E. M. Biometria de frutos e endocarpos de murici (Byrsonima verbascifolia Rich. Ex A. Juss.). Cerne, Lavras-MG, v. 12, n. 1, p.84-91, 2006.

HERBÁRIO INPA. Dulacia candida e Liriosma candida. 2015. Rede speciesLink. Disponível em: <http://www.splink.org.br>. Acesso em: 07 ago. 2015.

JOSHI, G.; PHARTYAL, S. S.; KHAN, M. R.; ARUNKUMAR, A. N. Recalcitrant morphological traits and intermediate storage behavior in seeds of Mesua ferrea, a tropical evergreen species. Seed Science and Technology, Bassersdorf, Suiça, v. 43, n. 1, p.121-126, 2015.

MONACHINO, J. A new Liriosma from Roraima. Brittonia, New York, USA, v.13, p.113-115, 1961. 
MOURA, R. C.; LOPES, P. S. N.; BRANDÃO JUNIOR, D. S.; GOMES, J. G.; PEREIRA, M. B. Biometria de frutos e sementes de Butia capitata (Mart.) Beccari (Arecaceae), em vegetação natural no norte de Minas Gerais, Brasil. Biota Neotropica, Campinas-SP, v. 10, n. 2, p.415-419, 2010.

NERY, M. C.; DAVIDE, A. C.; SILVA, E. A. A.; SOARES, G. C. M.; NERY, F. C. Classificação fisiológica de sementes florestais quanto a tolerância à dessecação e ao armazenamento. Cerne, Lavras-MG, v. 20, n. 3, p. 477-483, 2014.

NICKRENT, D. L.; MALÉCOT, V.; VIDAL-RUSSEL, R.; DER, J. P. A revised classification of Santalales. Taxon, Bratislava, Eslováquia, v. 59, n. 2, p. 538-558, 2010.

PEDRON, F.A., MENEZES, J.P. \& MENEZES, N.L. 2004. Parâmetros biométricos de fruto, endocarpo e semente de butiazeiro. Ciencia Rural, Santa Maria, v. 34, n. 2, p.585-586, 2004.

PRITCHARD, H. W. Classification of seed storage 'types' for ex situ conservation in relation to temperature and moisture. In: GUERRANT, E. O; HAVENS, K; MAUNDER, M. (Eds). Ex situ plant conservation: supporting species survival in the wild. Washington, DC: Island Press, p. 139-161. 2004.

PRITCHARD, H. W.; MOAT, J. F.; FERRAZ, J. B. S.; MARKS, T. R.; CAMARGO, J. L.; NADARAJAN, J.; FERRAZ, I. D. K. Innovative approaches to the preservation of forest trees. Forest Ecology and Management, Amsterdam, v. 333, p. 88-98, 2014.

RIBEIRO, J. E. L. S.; HOPKINS, M. J. G.; VICENTINI, A.; SOTHERS, C. A.; COSTA, M. A. S.; BRITO, J. M.; SOUZA, M. A. D.; MARTINS, L. H. P.; LOHMANN, L. G.; ASSUNÇÃO, P. A. C. L.; PEREIRA, E. C.; SILVA, C. F.; MESQUITA, M. R.; PROCÓPIO, L. C. Flora da Reserva Ducke: guia de identificação das plantas vasculares de uma floresta de terra-firme na Amazônia central. Manaus: INPA, 1999. $816 p$.

ROCHA, C. R. M.; COSTA, D. S.; MOVEMBRE, A. D. L. C.; CRUZ, E. D. Morfobiometria e germinação de sementes de Parkia multijuga Benth. Nativa, SinopMT, v. 2, n. 1, p.42-47, 2014.

ROYAL BOTANIC GARDENS KEW. Olacaceae. 2015. Seed Information Database (SID). Version 7.1. Disponível em: <http://data.kew.org/sid/>. Acesso em: 31 ago. 2015.

SACANDÉ, M.; JOKER, D.; DULLOO, M.E.; THOMSEN, K.A. Comparative storage biology of tropical tree seeds. Rome: IPGRI, 363p. 2004.

SANTANA, P. J. A. Maturação, secagem e armazenamento de sementes de espécies de Eugenia (Myrtaceae). 2007. 81 p. Dissertação (Mestrado em Biodiversidade Vegetal e Meio Ambiente) - Instituto de Botânica, São Paulo, 2007. 
SCALON, S. P. Q.; NEVES, E. M. S.; MASETO, T. E.; PEREIRA, Z. V. Sensibilidade à dessecação e ao armazenamento em sementes de Eugenia pyriformis Cambess. (uvaia). Revista Brasileira de Fruticultura, Jaboticabal-SP, v.34, n.1, p.269-276, 2012.

SILVA, K. S.; MENDONÇA, V.; MEDEIROS, L. F.; FREITAS, P. S. C.; GÓIS, G. B. de. Influência do tamanho da semente na germinação e vigor de mudas de jaqueira (Artocarpus heterophyllus Lam.). Revista Verde de Agroecologia e Desenvolvimento Sustentável, Pombal-PB, v. 5, n. 4, p. 217-221, 2010.

SILVA, K. B.; ALVES, E. U.; BRUNO, R. L. A.; CARDOSO, E. A. Tolerância à dessecação em sementes de Bunchosia armenica (Cav.) DC. Semina: Ciências Agrárias, Londrina-PR, v. 33, n. 4, p.1403-1410, 2012.

SOCOLOWSKI, F.; VIEIRA, D. C. M.; TAKAKI, M. Seed mass of Tecoma stans L. Juss. ex Kunth (Bignoniaceae): effects on emergence and seedling development under full sun and shade. Biota Neotropica, Campinas-SP, n. 11, v. 2, p.171-178, 2011.

SOUZA, V. C.; LORENZI, H. Botânica sistemática: guia ilustrado para identificação das famílias de Angiospermas da flora brasileira baseado em APG II. 3 ed. Nova Odessa: Instituto Plantarum, 2012. 768p.

TABARELLI, M. A.; VICENTE, D. C. A.; BARBOSA, D. Variation of seed dispersal spectrum of woody plants across a rainfall gradient in northeastern Brazil. Journal of Arid Environments, [S.I.], v. 53, p.197-210, 2003. 